\title{
SPLITTINGS OF CYCLIC $p$-ALGEBRAS
}

\author{
DAVID J. SALTMAN ${ }^{1}$
}

\begin{abstract}
In this note we investigate the finite p-groups appearing as Galois groups of maximal subfields of cyclic $p$-algebras and show that for a fixed cyclic $p$-algebra, all possible such groups appear.
\end{abstract}

In the following, a central simple algebra over a field of characteristic $p \neq 0$, and of degree a power of $p$, is called a $p$-algebra. A central simple algebra is called cyclic if it contains a maximal subfield which is a finite cyclic Galois extension of the center. We will assume that the reader is familiar with the theory of central simple algebras and the Brauer group.

If $G$ is a finite group, and $K$ is a field, we say $G$ appears over $K$ if there is a Galois extension $L$ of $K$ with Galois group $G(L / K)=G$. If, in addition, $A$ is a finite dimensional central simple algebra over $K$, we say $G$ appears over $K$ in $A$ if such an $L$ can be found which is also a maximal subfield of $A$. The purpose of this note is to prove the following theorem. In this theorem, as in the rest of the note, $K$ will always be a field of characteristic $p$.

THEOREM 1. If $A$ is a cyclic p-algebra of degree $p^{e}$ over $K$ and $G$ is a group of order $p^{e}$ appearing over $K$, then $G$ appears in $A$.

It is illuminating to take note of a result of Witt's at this point [5]. If $K$ is a field of characteristic $p$ and $\mathscr{P}: K \rightarrow K$ is the additive endomorphism $\mathscr{P}(k)$ $=k^{p}-k$, then $K / \mathscr{P}(K)$ can be considered as a vector space over the field $F(p)$ of $p$ elements. Let $N_{K}$ be $\operatorname{dim}_{F(p)} K / \mathscr{P}(K)$ if this is finite and $N_{K}=\infty$ if not. Then Witt showed that a finite $p$-group $G$ appears over $K$ if and only if $G$ is generated by $\leqslant N_{K}$ elements. We will make use later of the details of Witt's proof. Since we will show that the case $N_{K}$ finite is trivial, the main substance of what we will prove is the following theorem.

THEOREM $1^{\prime}$. If $K$ is a field of characteristic $p, N_{K}$ is infinite, and $A$ is a cyclic $p$-algebra over $K$ of degree $p^{e}$, then any group of order $p^{e}$ appears over $K$ in $A$.

The degree $p$ cyclic $p$-algebras are crucial to our proof. Let us recall some familiar facts about them. Any cyclic extension of $K$ of degree $p$ is of the form $K(\alpha)$ where $\mathscr{P}(\alpha) \in K$ but $\mathscr{P}(\alpha) \notin \mathscr{P}(K)$ and any such extension is cyclic of

Received by the editors March 16, 1976.

AMS (MOS) subject classifications (1970). Primary 16A40, 13A20; Secondary 12F10, 12F15.

Key words and phrases. Cyclic algebra, p-algebra, Galois group, Brauer group, characteristic $p$.

${ }^{1}$ The author was partially supported under N.S.F. Research Grant MPS72-04643. 
degree $p$. If $a=\alpha^{p}-\alpha$ and $L=K(\alpha)$ is cyclic of degree $p$ over $K$, then it can be shown that any $p$-algebra with maximal subfield $L$ is of the form $K\{x, y\} /\left(x^{p}-x-a, y^{p}-b, x y-y x-y\right)$ where $K\{x, y\}$ is the noncommutative polynomial ring in $x$ and $y, b \neq 0$ is in $K$. Call this algebra $[a, b)$ and call the images of $x$ and $y$ in $[a, b), \alpha$ and $\beta$ respectively. For any $a$ and $b \neq 0$ in $K,[a, b)$ is a cyclic $p$-algebra over $K$. If $a \notin \mathscr{P}(K)$, then $K(\alpha)$, the subalgebra generated by $\alpha$, is a cyclic field extension of $K$ of degree $p$. If $b \notin K^{p}, K(\beta)$ is a simple purely inseparable extension of $K$. In either case, the field mentioned is a maximal subfield of $[a, b)$. On the other hand, if $A$ is a $p$-algebra over $K$ with maximal subfield $K\left(b^{1 / p}\right)$ then $A \cong[a, b)$ for some $a \in K$. Finally, if $a \in \mathscr{P}(K)$ or $b \in K^{p},[a, b)$ is a trivial $p$-algebra, that is, a matrix algebra over $K$.

An elementary abelian $p$-group is a finite group of the form $C_{p} \oplus C_{p} \oplus \cdots$ $\oplus C_{p}$, where $C_{p}$ is the cyclic group of order $p$. If $L / K$ is a Galois extension with Galois group an elementary abelian $p$-group, then $L=L_{1} \otimes L_{2} \otimes \cdots \otimes L_{k}$ where $L_{i} / K$ is cyclic of degree $p$ and so $L_{i}=K\left(\alpha_{i}\right)$ with $\mathscr{P}\left(\alpha_{i}\right) \in K, \notin \mathscr{P}(K)$. The images of the $\mathscr{P}\left(\alpha_{i}\right)$ in $K / \mathscr{P}(K)$ must be linearly independent over $F(p)$. Conversely, if $a_{1}, a_{2}, \ldots, a_{k} \in K$ are linearly independent in $K / \mathcal{P}(K)$, then there is a unique $L=K\left(\alpha_{1}, \alpha_{2}, \ldots, \alpha_{k}\right)$, Galois over $K$ with group $C_{p} \oplus \cdots$ $\oplus C_{p}$ ( $k$ times), such that $\mathscr{P}\left(\alpha_{i}\right)=a_{i}$ and $L=K\left(\alpha_{1}\right) \otimes \cdots \otimes K\left(\alpha_{k}\right)$. The facts stated above are well known and can be found, for example, in [3].

The main technique used in this note is the manipulation of the algebras $[a, b)$. The first lemma, of this form, is the following.

LEMMA 2. If $K(\beta) \subseteq[a, b)$ is the $K$ subalgebra generated by $\beta$, and $c$ $=k_{0}+k_{1} \beta+\cdots+k_{p-1} \beta^{p-1}$ is in $K(\beta)$, then $[a, b) \cong[a+\delta(c), b)$ where $\delta(c)=k_{0}^{p}-k_{0}+k_{1}^{p} b+\cdots+k_{p-1}^{p} b^{p-1}$.

Proof. $[a, b)$ is generated by $\alpha+c$ and $\beta$, which satisfy the relations $\beta^{p}=b$ and $(\alpha+c) \beta-\beta(\alpha+c)=\beta$. We need only calculate $\mathscr{P}(\alpha+c)$ and show it equal to $\mathscr{P}(\alpha)+\delta(c)$.

Suppose $z$ has the property $[z, \beta]=\beta$. Then $\left[z, \beta^{i}\right]=i \beta^{i}$. Using [2, p. 187], we see that

$$
\left(z+k \beta^{i}\right)^{p}=z^{p}+\left(k \beta^{i}\right)^{p}+d_{z}^{p-1}\left(k \beta^{i}\right)=z^{p}+k^{p} b^{i}+(i)^{p-1} k \beta^{i},
$$

where $d_{z}$ is the inner derivation determined by $z$. Thus if $1 \leqslant i<p$,

$$
\mathscr{P}\left(z+k \beta^{i}\right)=\left(z+k \beta^{i}\right)^{p}-\left(z+k \beta^{i}\right)=\mathscr{P}(z)+k^{p} b^{i} .
$$

We compute $\mathcal{P}(\alpha+c)$ by induction. If $c=k_{0}$, clearly

$$
\mathscr{P}\left(\alpha+k_{0}\right)=\mathscr{P}(\alpha)+\mathscr{P}\left(k_{0}\right)=\mathscr{P}(\alpha)+\delta\left(k_{0}\right) .
$$

If $\quad 1 \leqslant i<p \quad$ and $c=k_{0}+k_{1}+\cdots+k_{i} \beta^{i}$, set $z=\alpha+k_{0}+\cdots$ $+k_{i-1} \beta^{i-1}$. Then by above, 


$$
\mathscr{P}(\alpha+c)=\mathscr{P}\left(z+k_{i} \beta^{i}\right)=\mathscr{P}(z)+k_{i}^{p} b^{i} .
$$

By induction, $\mathscr{P}(z)=\mathscr{P}(\alpha)+\delta\left(k_{0}+k_{1} \beta+\cdots+k_{i-1} \beta^{i-1}\right)$, and this does it. Q.E.D.

We can now deal with the case $N_{K}$ is finite, as well as the case $K$ is perfect. It is clear that a trivial $p$-algebra of degree $p^{e}$, i.e. a matrix algebra over $K$, contains as a maximal subfield any field extension of $K$ of degree $p^{e}$. If $K$ is perfect it is well known that all $p$-algebras are trivial and so Theorem 1 is obvious. Similarly, the next theorem will finish the case $N_{K}$ is finite.

THeOREM 3. If $N_{K}$ is finite, then any p-algebra over $K$ will be trivial.

Proof. It will suffice to show that the p-primary part of the Brauer group is zero. To show this, we need only show that any element of the Brauer group of exponent $p$ is trivial. By [1, pp. 108-109], any such element is similar to a product of algebras $\left[a_{i}, b_{i}\right)$. Thus it will suffice to show that such an algebra is trivial.

Suppose $c_{1}, c_{2}, \ldots, c_{n} \in K$ are representatives of a basis for $K / \mathcal{P}(K)$ over $F(p)$. We can assume $c_{i}=d_{i}^{p}, d_{i} \in K$, since modulo $\mathscr{P}(K), c_{i}$ and $c_{i}^{p}$ are the same. We can clearly assume $K$ is not perfect and therefore infinite. We must show any algebra $[a, b)$ is trivial and we can assume $b \notin K^{p}$. Call $K^{\prime}$ $=K\left(b^{1 / p}\right)=K(\beta) \subseteq[a, b)$. If $f \in K$, then $f c_{i}=\sum_{j} h_{i j} c_{j}+z$ where $z$ $\in \mathcal{P}(K)$ and $h_{i j} \in F(p)$. Consider the mapping sending $f$ to the $n \times n$ matrix $\left(h_{i j}\right) \in M_{n}(F(p))$. The quotient space $\left(K^{\prime}\right)^{p} / K^{p}$ is a vector space over $F(p)$ and so has some basis $\left\{\bar{b}_{i}\right\}_{i \in I}$. Choose representatives $b_{i}$ in $\left(K^{\prime}\right)^{p}$ of the $\bar{b}_{i}$. Since $K$ is infinite, $K^{p}$ is infinite and so $K^{p}$ is infinite dimensional as a vector space over $F(p)$. But $\left(K^{\prime}\right)^{p} / K^{p}$ is a nonzero vector space over $K^{p}$, and so it must be infinite dimensional over $F(p)$. That is, $I$ is infinite. Since $M_{p}(F(p))$ is finite, there are two representatives $b_{i}$ and $b_{j}$ with the same associated matrix in $M_{n}(F(p))$. Then $b^{\prime}=b_{i}-b_{j}$ has the properties $b^{\prime} c_{i} \in \mathcal{P}(K)$ for all $i$ and $b^{\prime} \notin K^{p}$. Hence $b^{*}=1+b^{\prime}$ has the properties $b^{*} c_{i}-c_{i} \in \mathcal{P}(K)$ for all $i$ and $b^{*} \notin K^{p}$. Since $b^{*} \in K^{\prime p}, K^{\prime}=K\left(b^{* 1 / p}\right)$, and $[a, b)=\left[a^{*}, b^{*}\right)$ for some $a^{*} \in K . a^{*}=h_{1} c_{1}+h_{2} c_{2}+\cdots+h_{n} c_{n}+z$ for some $h_{i}$ in $F(p)$ and $z$ in $\mathscr{P}(K)$, so if we call $\beta^{\prime}=b^{* 1 / p},\left(h_{i} d_{i} \beta^{\prime}\right)^{p}-h_{i} c_{i} \in \mathcal{P}(K)$. Thus by Lemma 2 , if $\quad c=h_{1} d_{1} \beta^{\prime}+h_{2} d_{2} \beta^{\prime}+\cdots+h_{n} d_{n} \beta^{\prime},\left[a^{*}, b^{*}\right) \cong\left[a^{*}+\delta(-c), b^{*}\right)=[z$, $b^{*}$ ) where $z \in \mathcal{P}(K)$. But this last algebra is trivial. Q.E.D.

This next lemma is the central fact of this note. The idea of this lemma is to change $b$ and then get " $a$ " to be a desired value.

Lemma 4. Suppose $A=[a, b)$ is a cyclic p-algebra over $K$ and $K^{\prime} \subseteq K$ is a subfield of $K$ such that $b \in K^{\prime}$ and $K / K^{\prime}$ is finite. Let $a^{\prime} \in K$ be arbitrary. Suppose $q$ is a power of $p$ strictly greater than the degree of $K$ over $K^{\prime}$. If $c \in K$ is such that $K^{\prime}(c)=K^{\prime}\left(c^{q}\right)=K, c \in K^{q}$ and $c^{q}=\sum k_{i} c^{i}$ where $k_{i} \in K^{\prime q}$, then $A \cong\left[a^{\prime \prime}, b+c\right)$ where $a^{\prime \prime}-a^{\prime} \in K^{\prime}$.

Proof. As usual, we can assume $b$ is not a $p$ th power in $K$. Let $d$ be the $p$ th root of $c$. Then if $\beta^{p}=b, K(\beta)=K(\beta+d)$ and so $A=\left[a_{1}, b+c\right)$ for some 
$a_{1} \in K$. Use $\beta+d$ to define the $\delta$ operator as in Lemma 2. Define $x \equiv y$ to mean that $x-y$ is in $\delta(K(\beta+d))$. By Lemma 2, it suffices to show that $a_{1} \equiv a^{\prime}+k^{\prime}, k^{\prime} \in K^{\prime}$. For this we need a sublemma.

Sublemma 5. Suppose $K^{\prime \prime}=K\left((b+c)^{1 / p}\right)$ and $(b+c)^{1 / p}$ is used to define the operator of Lemma 2. Then if $q$ is a power of $p$ greater than $n$, and $c, m \in K^{q}$, $m c^{n} \equiv(-1)^{n} m b^{n}$.

Proof. We will perform this proof by induction. Call $\beta=b^{1 / p}$ and $d=c^{1 / p} \in K$. Then $\delta\left((\beta+d) m^{1 / p}\right)=(b+c) m$, so $m c \equiv(-1) m b$. Assume the result for all $j<n$. If $n<p$, then considering $\delta\left(m^{1 / p}(\beta+d)^{n}\right)$, we get that

$$
m \sum_{i=0}^{n} b^{i} c^{n-i}\left(\begin{array}{c}
n \\
i
\end{array}\right) \equiv 0
$$

By induction, $(-1)^{n-i} m c^{n-i} c^{i} \equiv m c^{n-i} b^{i}$. Thus we have that

$$
m \sum_{i=0}^{n-1} b^{i} c^{n-i}\left(\begin{array}{l}
n \\
i
\end{array}\right) \equiv m c^{n}\left(\sum_{i=0}^{n-1}(-1)^{n-i}\left(\begin{array}{c}
n \\
i
\end{array}\right)\right) .
$$

But $\left(\sum_{i=0}^{n}(-1)^{n-i}\left(\begin{array}{c}n \\ i\end{array}\right)=(1-1)^{n}=0\right.$ so $\sum_{i=0}^{n-1}(-1)^{n-i}\left(\begin{array}{c}n \\ i\end{array}\right)=-(-1)^{n}$. Combining equations (1) and (2) gives our result for $n<p$. Now if $n \geqslant p$ then $n=r p+i$ for $r \geqslant 1$ and $0 \leqslant i<p$ so $m c^{n}=m c^{r p} c^{i}$. For any $x, x^{p} \equiv x$, so

$$
\begin{aligned}
m c^{r p} c^{i} & \equiv m^{1 / p} c^{r} d^{i} \equiv(-1)^{r} m^{1 / p} b^{r} d^{i} \quad \text { (by induction) } \\
& \equiv(-1)^{r p} m b^{r p} c^{i} \equiv(-1)^{r p} m b^{r p} b^{i}(-1)^{i} \quad \text { (by the } n<p \text { case). Q.E.D. }
\end{aligned}
$$

We will now finish the proof of Lemma 4. Since $a_{1}-a^{\prime}$ is in $K, a_{1}-a^{\prime}$ $=k_{0}+k_{1} c+\cdots+k_{n-1} c^{n-1}$ where $n$ is the degree of $K$ over $K^{\prime}$. It is now easy to see that $\left(a_{1}-a^{\prime}\right)^{q}=m_{0}+m_{1} c+\cdots+m_{n-1} c^{n-1}$ where all the $m_{i}$ are in $\left(K^{\prime}\right)^{q}$. Thus

$$
\begin{aligned}
a_{1}-a^{\prime} \equiv & \left(a_{1}-a^{\prime}\right)^{q} \\
\equiv & \left(\text { by the sublemma) } m_{0}+(-1) m_{1} b+(-1)^{2} m_{2} b^{2}\right. \\
& +\cdots+(-1)^{n-1} m_{n-1} b^{n-1}
\end{aligned}
$$

and this last expression is in $K^{\prime}$. Q.E.D.

Our reason for proving Lemma 4 was to prove the following result.

Lemma 6. Suppose $[a, b)$ is a cyclic p-algebra of degree $p$ over $K$ and $K^{\prime} \subseteq K$ is a subfield such that $K / K^{\prime}$ is finite and $b \in K^{\prime}$. If $a^{\prime}$ is in $K$ and separable over $K^{\prime}$, then there are $a^{\prime \prime}$ and $b^{\prime}$ in $K$ such that $[a, b) \cong\left[a^{\prime \prime}, b^{\prime}\right)$ and $a^{\prime}-a^{\prime \prime} \in K^{\prime}$.

Proof. Call $L$ the separable closure of $K^{\prime}$ in $K$. Since $[a, b) \cong\left[a+a^{p}\right.$ $-a, b)=\left[a^{p}, b\right)$, we can assume $a \in L$. Thus $[a, b)$ is defined over $L$ and we can assume $L=K$, that is, that $K / K^{\prime}$ is separable. Choose a $c \in K$ such that $K^{\prime}(c)=K$. Choose $p^{e}$ strictly greater than the degree of $K$ over $K^{\prime}$. By 
separability, $K=K^{\prime}\left(c^{p^{e}}\right)$, so $c^{p^{e}}=\sum k_{i} c^{i}, k_{i} \in K^{\prime}$. Thus using $c^{p^{e}}$ we can apply Lemma 4 . Q.E.D.

We will prove the main result, Theorem $1^{\prime}$, in two steps. The first step is embodied in the next result. As a preliminary, we must mention a theorem of Albert's [1, p. 107]. A $p$-algebra $A$, of degree $p^{n}$ over its center $K$, is cyclic if and only if it is split by a simple purely inseparable extension $K^{\prime}$ of degree $p^{f}, f \leqslant n$. If $K$ is not perfect, we can assume $f=n$. Thus, over a nonperfect field, a $p$-algebra is cyclic if and only if it has a simple purely inseparable maximal subfield.

Proposition 7. Let $K$ be a field of characteristic $p$ with $N_{K}$ infinite, and $A$ a cyclic p-algebra of degree $p^{e}$ over $K$. Then the elementary abelian p-group of order $p^{e}$ appears over $K$ in $A$.

Proof. As usual, we may assume $K$ is not perfect. Thus $A$ contains a maximal subfield $L=K(\beta)$ such that $\beta^{p^{e}} \in K$ but $\beta^{p^{e-1}} \notin K$. We now proceed by induction on $e$. If $e=1$, the result is trivial. Assume it for $e-1$. Call $L^{\prime}=K\left(\beta^{p^{e-1}}\right)$ and $A^{\prime}=A^{L^{\prime}}$, the centralizer of $L^{\prime}$ in $A$. Then $A^{\prime}$ is central simple over $L^{\prime}$ of degree $p^{e-1}$ with maximal subfield $L$, and thus $A^{\prime}$ is also cyclic. The $p$-power map induces an isomorphism from $L^{\prime} / \mathscr{P}\left(L^{\prime}\right)$ to $K / \mathcal{P}(K)$, and so $N_{L^{\prime}}=N_{K}$. By induction, $A^{\prime}$ contains a maximal subfield $M^{\prime}$ Galois over $L^{\prime}$ with Galois group the elementary $p$-group of order $p^{e-1}$. Since $L^{\prime} / K$ is purely inseparable, $M^{\prime}=M \otimes L^{\prime}$ where $M / K$ is Galois with the same Galois group as $M^{\prime} / L^{\prime}$. Thus we can write $M=K\left(\alpha_{1}, \alpha_{2}, \ldots, \alpha_{e-1}\right)$ where $\mathscr{P}\left(\alpha_{i}\right)=a_{i} \in K$ and the $a_{i}$ 's are linearly independent in $K / \mathcal{P}(K)$. $M$ is a subfield of $A$ of degree $p^{e-1}$ and so $A^{*}=A^{M}$ is a $p$-algebra of degree $p$. Since $L^{\prime}$ commutes with $M, L^{\prime} \subseteq A^{*}$. Thus for some $a \in M, A^{*} \cong[a, b)$ where $b=\beta^{p^{e}} \in K$. By Lemma 6, choosing $a^{\prime}=0, A^{*}=\left[a^{*}, b^{*}\right)$ where $a^{*} \in K$. Call $M^{*}=M\left(\alpha^{*}\right)$, where $\alpha^{*} \in A^{*}$ and $\mathscr{P}\left(\alpha^{*}\right)=a^{*}$. If $a^{*}$ is linearly independent of the $a_{i}$ 's in $K / \mathscr{P}(K)$, then $M^{*}$ is Galois over $K$ with the required group. If not, then $a^{*} \in \mathscr{P}(M)$ and so $A^{*}$ is trivial. Thus $A^{*}$ contains any extension of degree $p$ and the result is easy using the fact that $N_{K}$ is infinite. Q.E.D.

We are now ready to deal with the case of arbitrary $p$-groups. If $G$ is a finite $p$-group, define $G^{*}$ to be the subgroup generated by the commutator subgroup $G^{\prime}$ of $G$ and the set $G^{p}=\left\{g^{p} \mid g \in G\right\}$. Then $G / G^{*}$ is an elementary abelian $p$-group and by Burnside's basis theorem (e.g. [4, p. 161]), the minimal number of generators of $G$ and $G / G^{*}$ are the same. The essence of the result of Witt's mentioned at the beginning was a proof of his that $G$ appears over $K$ if and only if $G / G^{*}$ does. We will review his proof and show that it can be performed inside a fixed cyclic $p$-algebra.

Call $Z=Z(G)$ the center of $G$. We assume $G^{*} \neq(e)$. Then $Z \cap G^{*} \neq(e)$ (e.g. [4, p. 139]). Choose $g \in Z \cap G^{*}$ of order $p$ and call $H$ the subgroup generated by $g$. We have the extension $0 \rightarrow H \rightarrow G \rightarrow G / H \rightarrow 0$. Choose representatives $u_{\sigma}$ for each $\sigma \in G / H$. As usual, define the group cohomology 
2-cocycle $g_{\sigma, \tau}$ by the relation $u_{\sigma} u_{\tau}=g_{\sigma, \tau} u_{\sigma \tau}$. Being a 2-cocycle, $g_{\sigma, \tau} g_{\rho, \sigma \tau}$ $=g_{\rho, \sigma} g_{\rho \sigma, \tau}$. Since $H$ is central in $G, u_{\sigma} g^{\prime}=g^{\prime} u_{\sigma}$ for all $g^{\prime} \in H$. These conditions completely determine $G$ given $H$ and $G / H$.

Suppose we have an extension $L / K$ with Galois group $G / H$. Define $\chi \in \operatorname{Hom}(H, F(p)) \quad$ by $\chi(g)=1$. Then $\chi\left(g_{\sigma, \tau}\right)+\chi\left(g_{\rho, \sigma \tau}\right)=\chi\left(g_{\rho, \sigma}\right)$ $+\chi\left(g_{\rho \sigma, \tau}\right)$. Thus $\chi\left(g_{\sigma, \tau}\right)$ is a 2 -cocycle of $G / H$ into $L^{+}$. Since $H^{2}\left(G / H, L^{+}\right)$ $=0$, there is a $d: G / H \rightarrow L^{+}$such that $\chi\left(g_{\sigma, \tau}\right)=d_{\sigma}+\sigma d_{\tau}-d_{\sigma \tau}$. Since $\chi\left(g_{\sigma, \tau}\right) \in F(p)$, we have that $\mathscr{P}\left(d_{\sigma}\right)+\sigma \mathcal{P}\left(d_{\tau}\right)-\mathscr{P}\left(d_{\sigma \tau}\right)=0$. That is, $\mathscr{P}\left(d_{\sigma}\right)$ is a 1 -cocycle. But $H^{1}\left(G / H, L^{+}\right)=0$, so there is a $\gamma \in L$ such that $\mathscr{P}\left(d_{\sigma}\right)$ $=(\sigma-1) \gamma$. Define $L^{\prime}=L(\alpha)$, where $\mathscr{P}(\alpha)=\gamma$. It is shown in [5] that $L^{\prime} / K$ is a Galois field extension with Galois group $G$. Note that if $\gamma$ is changed to $\gamma+k$, with $k \in K$, the result still holds.

We are finally ready to prove Theorem $1^{\prime}$. Suppose $A$ is a cyclic $p$-algebra of degree $p^{e}$ over a field $K$ with $N_{K}=\infty$, and $G$ is a group of order $p^{e}$. We proceed by induction on $e$. As usual, we can assume $K$ is not perfect. Define $G^{*}, H, u_{\sigma}$, and $g_{\sigma, \tau}$ as above. By Proposition 7 we can assume $G^{*} \neq(e)$. Suppose $L=K(\beta)$ is a simple purely inseparable maximal subfield of $A$ of degree $p^{e}$ and, as before, define $L^{\prime}=K\left(\beta^{p^{e-1}}\right)$ and $A^{\prime}=A^{L^{\prime}}$. Then $A^{\prime}$ is cyclic of degree $p^{e-1}$ over $L^{\prime}$ and by induction contains a maximal subfield $M^{\prime}$ Galois over $L^{\prime}$ with Galois group $G / H$. As before, $M^{\prime}=M \otimes L^{\prime}$ where $M / K$ is Galois with group $G / H$. Using $M$ we can define $\chi, d_{\sigma}$, and $\gamma \in M$ as above. Call $A^{*}=A^{M}$, so $A^{*} \cong[a, b)$ with $b \in K$. By Lemma $6, A^{*} \cong\left[a^{*}, b^{*}\right)$ with $a^{*}-\gamma \in K$. If $\alpha^{*} \in A^{*}$ is such that $\mathscr{P}\left(\alpha^{*}\right)=A^{*}$, then $M\left(\alpha^{*}\right)$ is Galois over $K$ with group $G$. This completes the theorem and the note.

\section{BIBLIOGRAPHY}

1. Adrian Albert, Structure of algebras, Amer. Math. Soc. Colloq. Publ., vol. 24, Amer. Math. Soc., Providence, R.I., 1961. MR 23 \# A912.

2. Nathan Jacobson, Lie algebras, Interscience, New York, 1962. MR 26 \#1345.

3. Paul J. McCarthy, Algebraic extensions of fields, Blaisdell, Waltham, Mass., 1966. MR 33 \#5612.

4. W. R. Scott, Group theory, Prentice-Hall, Englewood Cliffs, N.J., 1964. MR 29 \#4785.

5. E. Witt, Konstruktion von galoisschen Körpern der Charakteristik p zu vorgegebner Gruppe de Ordnung $p^{f}$, J. für Math. 174 (1936), 237-245.

Department of Mathematics, University of Chicago, Chicago, Illinois 60637 